\title{
Characterization of Novel 8T SRAM with Low Leakage and Optimized Area
}

\author{
Kazi Fatima Sharif and Satyendra N. Biswas* \\ Department of Electrical and Electronic Engineering, Ahsanullah University of Science and Technology Dhaka -1208, Bangladesh. \\ *Corresponding Author, email: sbiswas@linuxmail.org / sbiswas.eee@aust.edu).
}

\begin{abstract}
The fast evolution of battery functioned devices has caused approaches for decreasing power consumption in the memories is substantial. In this paper, a new proposal of SRAM with 8 transistors $(8 T)$ has been designed and also the cell itself is tested for its unique data overwriting and read propagation delays around $13.33 \%$ (read ' 1 ') and $3.58 \%$ (read ' 0 ') less compared to a conventional model. As the technology is attenuating, cell stability and increasing noise margin have become two crucial topics for the design metrics of SRAM, where our proposed cell appears with great stability on low voltage operation. Widespread simulation results authenticate the cogency and competency of the proposed 8T SRAM model using Cadence and $45 \mathrm{~nm}$ predictive technology model (PTM).
\end{abstract}

Keywords - Low power SRAM; memory cell; nMOS; successive read; static noise margin.

\section{INTRODUCTION}

The high stability and low power have been the main components of SRAM architecture in the past decade. It has been estimated that almost $94 \%$ of total die area in a chip can be occupied by Static random access memory (SRAM) in upcoming nanoscale technology future [1]. Device scaling caused several challenges while designing SRAM cell. Such as applying low threshold voltage in thin gate oxide not only affects the data stability but also increases the energy consumption [2]. Also, the fundamental parameters like, dopant and oxide thickness fluctuation, line edge roughness additionally damages the constancy of SRAM [3-5].

The conventional 6 transistor (6T) SRAM with single end suffers from write delay [6]. Another 6T SRAM with low power can gain back the time delay but hampers in data stability [7]. Using the $200 \mathrm{mV}$ sub-threshold voltage performed comparatively better in another design [8]. To overcome the drawbacks of conventional 6T SRAM by accumulating additional transistor researcher have introduced several new SRAM designs which affected the increased overhead area around 30\% [9]. A conventional 7T SRAM performs better instability when a low supply voltage is provided [10]. An alternative design of 7T SRAM with a $360 \mathrm{~nm}$ width of nMOS in parallel with a $240 \mathrm{~nm}$ width of access transistor not only reduced the leakage current to pass in static mode but also improves the write time delay [11]. Comparing with 6T and 7T SRAM conventional 8T SRAM cell occupies much space in a system.
An Ultra Dynamic Voltage Scaling (UDVS) technique was used to minimize energy consumption by adjusting supply voltage in 8T SRAM model [12].

A different 8T SRAM design consists of a single column based dynamic supply voltage technology recovers the data read and write stability [13]. Furthermore, D. A. Tuan et al, introduced a disturb-decouple differential 8T SRAM cell to eliminate the half access concerns related with both static Noise Margin (SNM) and the Write Trip Point (WTP) can be improved alongside [14].

Again, a cross point 8T-SRAM with a negative bias technique has been designed by M. Yabuuchi, et. al. that realizes both large cell stability and write ability under low operating voltage by reducing active power by $66 \%$, compared to the conventional 6T SRAM [15]. However the design amplified almost $2 \mathrm{X}$ higher noise margin than the conventional.

The present proposal shows a new design of 8T SRAM cell which contains all nMOS transistors replacing pMOS transistors associated with conventional 8T SRAM model. With this replacement, the proposed cell performs better in time delay, power consumption and noise margin. The overhead area also shrinks. The rest of paper is organized as follows: section II presents the proposed cell. In section III, simulation results are discussed thoroughly while section IV concludes with the results.

\section{PROPOSED 8T SRAM CELL}

Figure 1 shows the proposed SRAM circuit of 8 transistors. It was constructed with $8 \mathrm{nMOS}$ transistor. The pMOS of conventional 6T SRAM are replaced by two nMOS (M1, M2) transistors, where the gates of those two transistors are connected with two storage nodes Q and QB respectively. The source of M3 and M4 transistors are connected with the ground and the drain of those two transistors are connected with the source of M1 and M2 respectively. The voltage source is connected with the drain of M1 and M2. The other two transistors M7 and M8 are also new inclusion to this proposed SRAM compared to conventional 6T SRAM. Basically SRAM has three operational modes: write operation, hold or static operation and read operation. 
The supply voltage we used as Vdd varies from $1 \mathrm{v}$ to $0.7 \mathrm{v}$ for different performance analysis.

\section{A. Write '1' operation:}

Data is delivered through BL and BLB with the help of access transistors M5, M6 and write word line (WRL). Data is then stored in Q and QB storage nodes. For Instance, after a write operation, if $\mathrm{BL}$ is provided ' 1 ' the storage node $\mathrm{Q}$ will store ' 1 ' and the complimentary storage node QB stores ' 0 '. The transistors M1 and M4 will be ON, however transistors M2 and M3 will be OFF. The current from source voltage Vdd will pass through $\mathrm{M} 1$ and helps to store the data in Q node. There will also create a discharge path connected to ground through M4 transistor which helps to store the data in QB node. So the data in both storage nodes remains unchanged.

\section{B. Write '0' operation:}

Similarly, while writing ' 0 ', BL is provided by data ' 0 ' which will store ' 0 ' in storage node $\mathrm{Q}$ and complimentary storage node QB will store ' 1 '. The state of transistors get inverted which means M1 and M4 transistor will be OFF nevertheless M2 and M3 transistors will be ON. Ground node will connect with node $\mathrm{Q}$ and supply voltage Vdd will connect with QB. So the data stored in storage node is safely stored and about to be read in read mode.

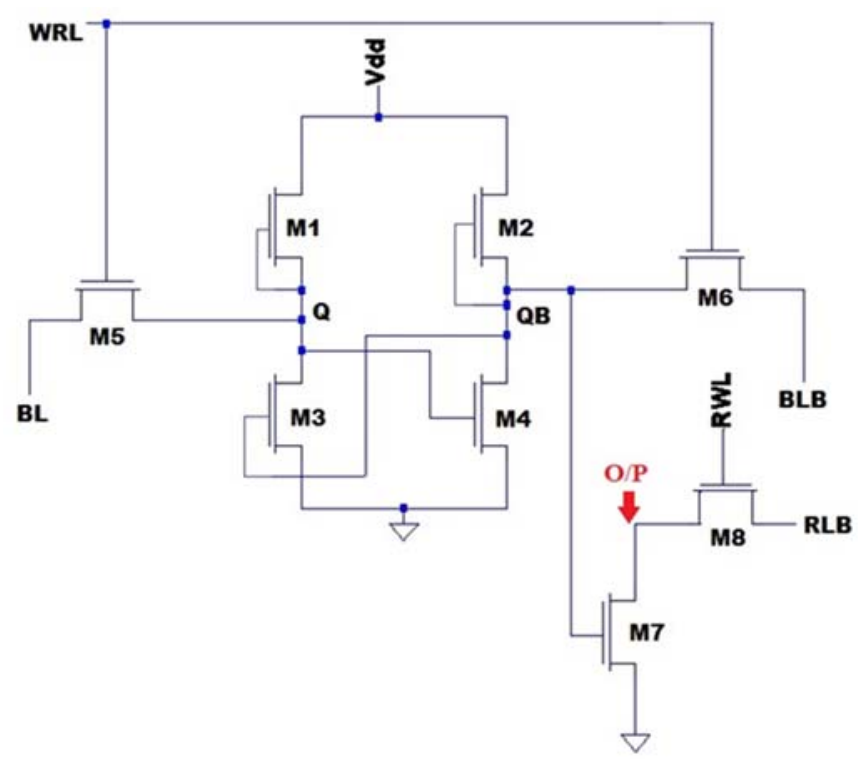

Fig. 1. Schematic design of proposed 8T SRAM cell

\section{Read operation:}

RBL and RWL is highly charged which makes transistor M8 on. The gate of the transistor M7 is connected with storage node QB. So the state of QB will handle the operation of M7 transistor. If we want to read ' 1 ', storage node $\mathrm{Q}$ should store ' 1 ' and complimentary storage node QB should store ' 0 ' which turns off the $M 7$ transistor. So in output node $(\mathrm{O} / \mathrm{P})$ we will find our desired read data ' 1 '. In the same way when we want to read '0', Q should store '0' and QB should store '1', which turns on the M7 transistor. The output node $(\mathrm{O} / \mathrm{P})$ gets connected with ground through M7 transistor and will show us a discharged data equivalent to ' 0 '. Thus we get our desirable read data.

\section{Simulations And Results}

The focus of this work is on designing memory circuit in nanoscale technology overcoming the previous challenges. Extensive simulation experiments have done using Cadence Design Systems and LTSpice by PTM transistor model. This section elaborately describes the performance comparison between previous conventional SRAM models and proposed 8T SRAM Cell on the basis of time delay, static noise margin and power consumption. The width of pull-up (PU) transistors M1 and M2 are designed smaller than the width of access (AU) transistors M5 and M6 which are also smaller than the width of pull-down (PD) transistors M3 and M4. Wpu, Wau and Wpd are chosen as $32 \mathrm{~nm}, 45 \mathrm{~nm}$ and $65 \mathrm{~nm}$ [16].

\section{A. Write '0' overwriting ' 1 ' operation:}

Single ended and low power 6T SRAM reduces the write time access which tends to suffer from data write ability [22]. Write ability of a SRAM cell is determined by pull-up ratio (PR) or $\gamma$ ratio. Commonly, $\mathrm{PR} \leq 1.8$ is required to uphold good write ability [17].

$$
\text { Pull-up ratio, } \gamma=\frac{(\mathrm{W} / \mathrm{L}) \text { access transistors }}{(\mathrm{W} / \mathrm{L}) \text { pull up transistors }}=\frac{45 \mathrm{~nm}}{32 \mathrm{~nm}}=1.4
$$

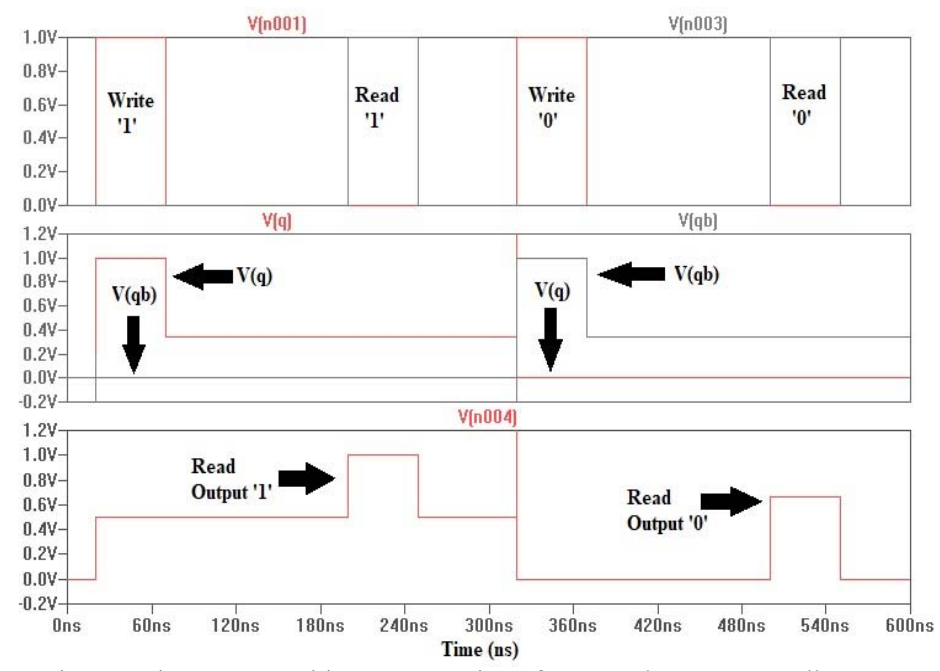

Fig. 2. Write '0' overwriting ' 1 ' operation of proposed 8T SRAM cell.

Figure 2 shows the result of storing ' 0 ' after overwriting stored data ' 1 '. Here there are two write and read cycle. In write ' 1 ' the data stored data $\mathrm{V}(\mathrm{Q})$ is ' 1 ' as a consequence we are getting the read output result ' 1 ' in read cycle ' 1 '. But in write ' 0 ' cycle the data is flipped at $320 \mathrm{~ns}$ where stored data $\mathrm{V}(\mathrm{QB})$ is storing ' 0 ' as a consequence the read output of read cycle ' 0 ' is showing the lowest data around $0.5 \mathrm{~V}$. This significances that the proposed 8T SRAM cell is highly capable of writing and storing random data.

\section{B. Successive read operation:}

Inaccuracy free successive read operation is one vital characteristic for memory circuit. The SRAM cell is most susceptible to noise throughout read operation since the storage node is storing ' 0 ' which will rise to a higher voltage than ground by reason of voltage dividing outcome between the access transistors. Cell ratio (CR) or $\beta$ of SRAM is required to avoid read failure. Cell ratio controls how high the ' 0 ' storing node escalates during read access. 
[17].

Cell ratio, $\beta=\frac{(\mathrm{W} / \mathrm{L}) \text { pull down transistors }}{(\mathrm{W} / \mathrm{L}) \text { access transistors }}=\frac{65 \mathrm{~nm}}{45 \mathrm{~nm}}=1.4$

For writing data ' 1 ' the storage node is storing $\mathrm{V}(\mathrm{Q})$ ' 1 ' and complimentary storage node $\mathrm{V}(\mathrm{QB})$ is storing ' 0 '. Figure 3, reports that after writing ' 1 ' for three consecutive read cycle (read mode ' 1 ', read mode ' 2 ' and read mode ' 3 ') we are obtaining read output ' 1 '. The voltage difference between two storage nodes remains almost same during successive read period.

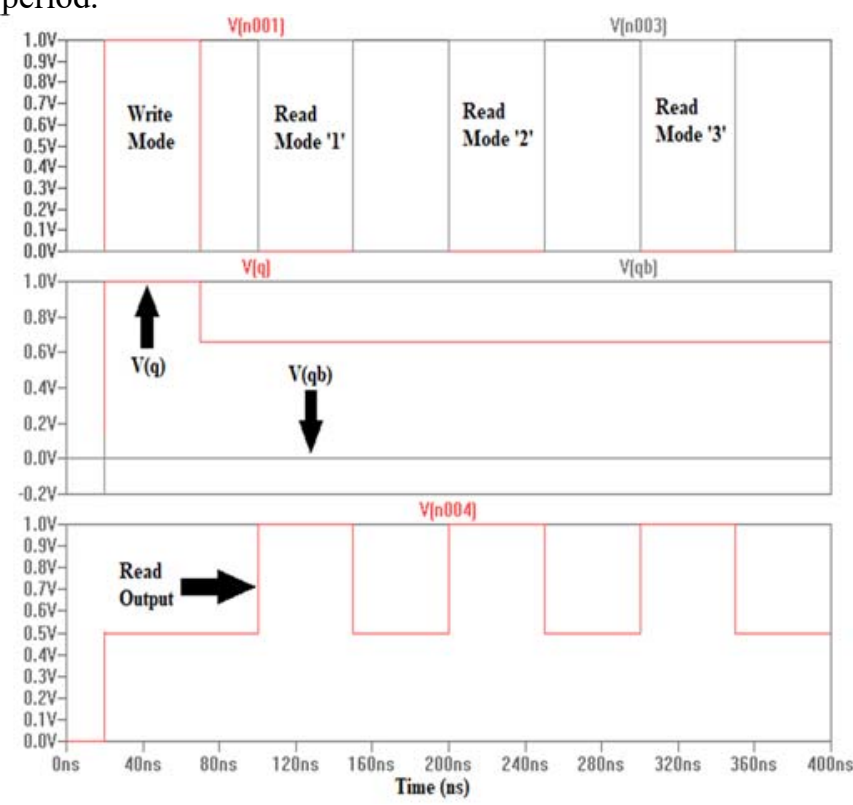

Fig.3. Successive read operation of proposed 8T SRAM cell.

\section{Time delay calculation:}

In figure 4 comparison of write and read time delay for both ' 1 ' and ' 0 ' has been revealed between conventional $8 \mathrm{~T}$ and proposed 8T SRAM cell. Here both the models are designed in CMOS $45 \mathrm{~nm}$ technology. From figure 4 we can settle that while writing the data proposed model spends almost $28.57 \%$ more time than conventional $8 \mathrm{~T}$ model. But even though reading the data proposed model employs less time delay than conventional 8T SRAM, which is $13.33 \%$ (read ' 1 ') and $3.58 \%$ (read ' 0 ').

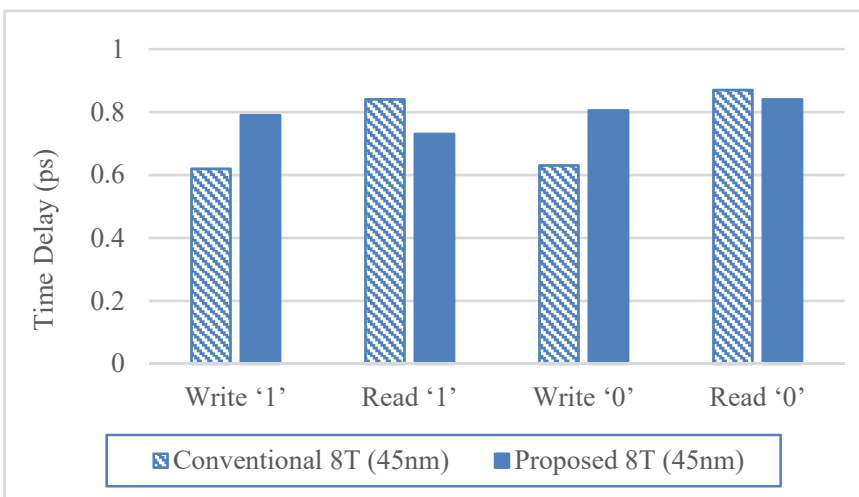

Fig.4. Time delay calculation between conventional 8T SRAM and proposed ST SRAM.

Nevertheless the write time of proposed 8T SRAM was not up to the mark while relating it with conventional 8T SRAM, but if we equate the same time delay with conventional $6 \mathrm{~T}$ replaced by conventional $8 \mathrm{~T}$, our proposed $8 \mathrm{~T}$ SRAM shows an admirable result. Figure 5 illustrates writing data ' 1 ' for conventional $6 \mathrm{~T}$ the $\mathrm{Q}$ store node takes almost $0.8 \mathrm{ps}$ to reach $80 \%$ of peak value. However, the proposed $8 \mathrm{~T}$ takes around $0.6 \mathrm{ps}$ to reach the $80 \%$ of the peak $1 \mathrm{~V}$ (figure 6 ).

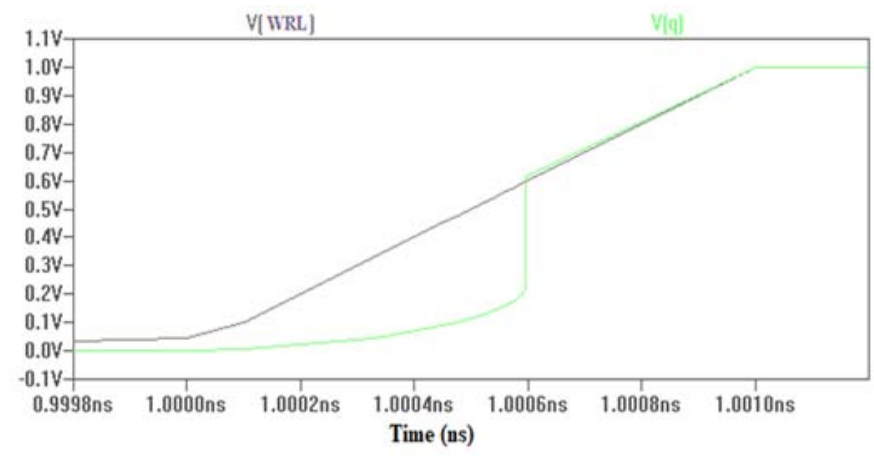

Fig. 5. Write '1' time delay of conventional 6T (45nm) SRAM.

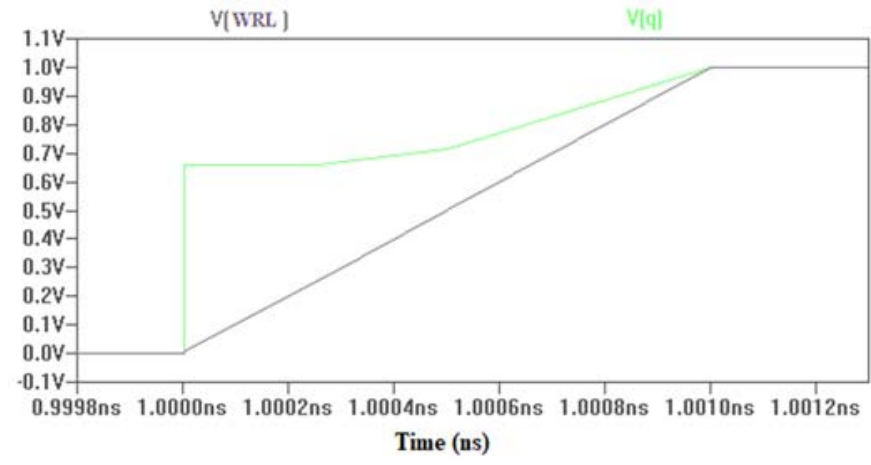

Fig. 6. Write ' 1 ' time delay of proposed $8 \mathrm{~T}$ ( $45 \mathrm{~nm}$ ) SRAM.

In order to compare the time delay of the proposed model (implemented in $45 \mathrm{~nm}$ technology) with the conventional $8 \mathrm{~T}$ SRAM [18], their model has been redesigned with $45 \mathrm{~nm}$ technology. The calculated results along with the original results of the reference paper [18] are depicted in table I. The proposed $8 \mathrm{~T}$ SRAM cell with $45 \mathrm{~nm}$ length demonstrates better performance in terms of time delay for both writing and reading operations.

Table I

COMPARISON OF TIME DELAY For DifFERENT MODEL

\begin{tabular}{lcc}
\hline \hline Parameters & Write & Read \\
\hline Proposed 8T SRAM (45nm) & $0.79 \mathrm{ps}$ & $0.73 \mathrm{ps}$ \\
8T SRAM (45nm) [18] & $0 / 88 \mathrm{ps}$ & $0.82 \mathrm{ps}$ \\
8T SRAM (180nm) [18] & $63.27 \mathrm{ps}$ & $175.92 \mathrm{ps}$
\end{tabular}

\section{Power consumption:}

The dynamic power dissipation can be minimized by scaling the supply voltage. Nevertheless scaling the supply voltage hampers the noise margin of the system [20]. Improving the power consumption has been a tough challenge for researcher. Table II shows the comparison of power consumption among different models of SRAM, where the conventional 6T SRAM [19] and 8T SRAM [18] are redesigned in $45 \mathrm{~nm}$ technology like the proposed model. The 
conventional 8T SRAM model which was simulated with the length of $45 \mathrm{~nm}$ is compared with its own model of $180 \mathrm{~nm}$ technology [18] in Table II alongside with the proposed 8T SRAM. Here with the growth of length, consumption of power increases. Our proposed 8T SRAM (45nm) model transports lowest power consumption $1.89 \mathrm{pW}$, which is $66.90 \%$ less than conventional 8T SRAM of $45 \mathrm{~nm}$ and $182.18 \%$ fewer than the conventional 8 T SRAM of $180 \mathrm{~nm}$.

Table II

POWER CONSUMPTION AMONG DifFERENT MODEL OF SRAM

\begin{tabular}{lc}
\hline \hline Parameters & Write power consumption \\
\hline 8T SRAM (180nm) [18] & $40.54 \mathrm{pW}$ \\
6T SRAM (45nm) [19] & $7.5 \mathrm{pW}$ \\
8T SRAM (45nm) [18] & $3.79 \mathrm{pW}$ \\
Proposed 8T SRAM (45nm) & $1.89 \mathrm{pW}$ \\
\hline \hline
\end{tabular}

The static (leakage) power consumption has twisted undesirable effects on SRAM devices. By abating the leakage power the data can be safe while reading mode. From figure 7, we can see that the proposed 8T SRAM shows approximately $1.9 \mathrm{pW}$ less power ingestion than conventional $8 \mathrm{~T}$

SRAM.

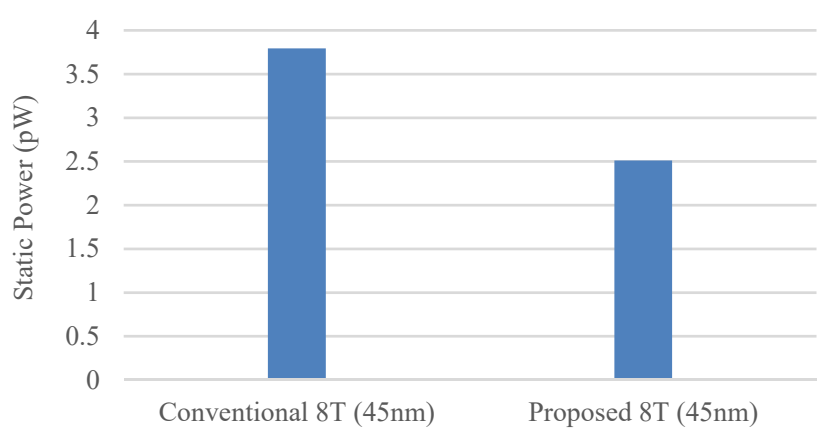

Fig. 7. Static power dissipation between conventional 8T SRAM and proposed 8T SRAM.

\section{E. Static noise margin:}

SNM measures the amount of voltage noise required at the storage nodes of a SRAM to flip the cell's contents [20]. By

Table III

Operating Mode Of Leakage Current At The Static Mode Of Proposed 8T SRAM

\begin{tabular}{|c|c|c|c|c|}
\hline Device & $\mathrm{V}_{\mathrm{GS}}$ & $\bar{V}_{\mathrm{DS}}$ & State & $\begin{array}{c}\text { Operating } \\
\text { mode }\end{array}$ \\
\hline M1 & $\mathrm{V}_{\mathrm{Q}}=0.99978 \mathrm{~V}$ & $\mathrm{Vdd}-\mathrm{V}_{\mathrm{Q}}=1 \mathrm{~V}-0.99978 \mathrm{~V} \approx 0 \mathrm{~V}$ & $\mathrm{Vgs}=0$ & cutoff \\
\hline M2 & $\mathrm{V}_{\mathrm{QB}}=15.809176 \mathrm{nV} \approx 0 \mathrm{~V}$ & $\mathrm{Vdd}-\mathrm{V}_{\mathrm{QB}}=1 \mathrm{~V}-15.809176 \mathrm{nV} \approx 1 \mathrm{~V}$ & $\mathrm{~V}_{\mathrm{DS}}>\mathrm{Vgs}-\mathrm{Vt}$ & saturation \\
\hline M3 & $\mathrm{V}_{\mathrm{QB}}=15.809176 \mathrm{nV} \approx 0 \mathrm{~V}$ & $\mathrm{~V}_{\mathrm{Q}}=999.74865 \mathrm{mV}$ & $\mathrm{Vgs}<\mathrm{Vt}$ & cutoff \\
\hline M4 & $\mathrm{V}_{\mathrm{Q}}=0.99978 \mathrm{~V}$ & $\mathrm{~V}_{\mathrm{QB}}=15.809176 \mathrm{nV} \approx 0 \mathrm{~V}$ & $\mathrm{Vds}<\mathrm{Vgs}-\mathrm{Vt}$ & Linear \\
\hline M5 & $\mathrm{V}_{\mathrm{WRL}}-\mathrm{V}_{\mathrm{Q}}=0 \mathrm{~V}-0.99978 \mathrm{~V}=-0.99978 \mathrm{~V}$ & $\mathrm{~V}_{\mathrm{BL}}-\mathrm{V}_{\mathrm{Q}}=1 \mathrm{~V}-0.99978 \mathrm{~V}=2.2 \mathrm{e}-04 \mathrm{~V}$ & $\mathrm{Vds}>$ vgs or, $\mathrm{Vds}+\mathrm{Vt}>\mathrm{Vgs}$ & saturation \\
\hline M6 & $\mathrm{V}_{\mathrm{WRL}}-\mathrm{V}_{\mathrm{QB}}=0-0 \mathrm{~V}(15.809176 \mathrm{nV})=0 \mathrm{~V}$ & $\mathrm{~V}_{\mathrm{BLB}}-\mathrm{V}_{\mathrm{QB}}=0 \mathrm{~V}-0 \mathrm{~V}(15.809176 \mathrm{nV})=0 \mathrm{~V}$ & $\mathrm{Vgs}<\mathrm{Vt}$ & cutoff \\
\hline M7 & $\mathrm{V}_{\mathrm{QB}}=15.809176 \mathrm{nV} \approx 0 \mathrm{~V}$ & $\mathrm{~V}_{\mathrm{O} / \mathrm{P}}=13.1476 \mathrm{uV}$ & $\mathrm{Vgs}<\mathrm{Vt}$ & cutoff \\
\hline M8 & $V_{R W L}-V_{O / P}=0-13.1476 \mathrm{uV}=-13.1476 \mathrm{uV}$ & $\mathrm{V}_{\mathrm{RLB}}-\mathrm{V}_{\mathrm{O} / \mathrm{P}}=1 \mathrm{~V}-13.1476 \mathrm{uV}=0.999 \mathrm{~V}$ & Vds $>$ Vgs & Saturation \\
\hline
\end{tabular}

introducing noise $\left(\mathrm{V}_{\mathrm{N}}\right)$ in storage node $\mathrm{Q}$ or Qbar shown in figure 8 , the data gets un-established and the noise margin is detected.

Figure 9, plots the VTC (voltage transfer characteristics) and inverse VTC of two inverters (M1, M3 and M2, M4) consequtively at nominal temperature $27^{\circ} \mathrm{C}$. The resulting two lobed curve is called 'butterfly curve'. The length of the side of the largest square that can be drawn inside the lobes of the butterfly curves, is how the SNM is measured [21].

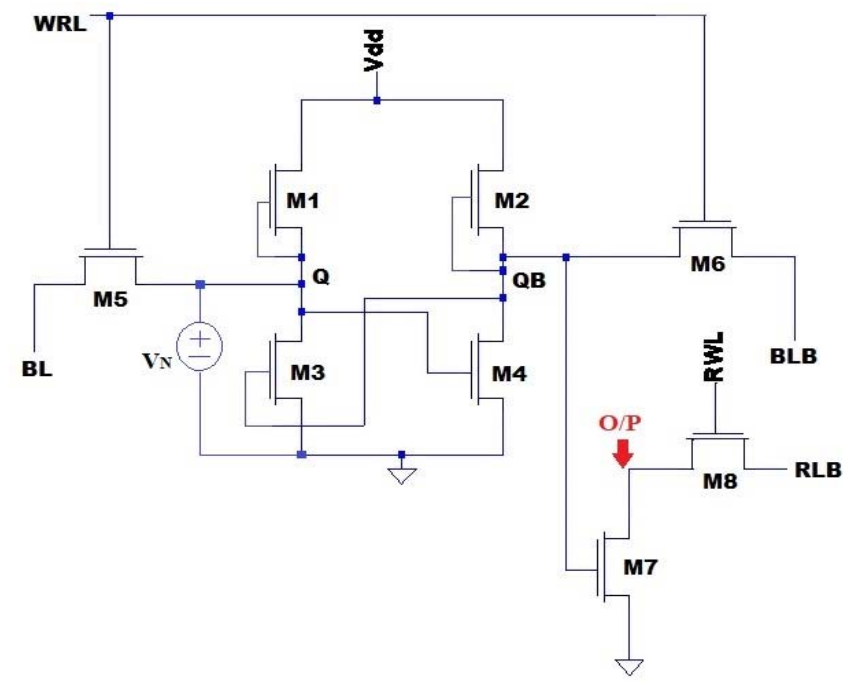

Fig. 8. Scametic of proposed 8T SRAM cell showing noise voltage source for finding SNM.

SNM for a SRAM with ideal VTCs is still inadequate to $\frac{\mathrm{Vdd}}{2}$ because of the two sides of the butterfly curve. Figure 9, plots SNM of proposed cell by varying different supply Vdd $(0.7 \mathrm{~V}, 0.8 \mathrm{~V}, 0.9 \mathrm{~V}$ and $1 \mathrm{~V})$. For $0.7 \mathrm{~V}$ and $0.8 \mathrm{~V}$ as supply the largest square of butterfly curve show SNM around $0.53 \mathrm{~V}$ and $0.68 \mathrm{~V}$ whereas $0.9 \mathrm{~V}$ and $1 \mathrm{~V}$ as supply the largest square of butterfly curve show SNM around $0.75 \mathrm{~V}$ and $0.9 \mathrm{~V}$ (figure $10)$. It can be stated that with the escalation of supply voltage noise margin rises. 


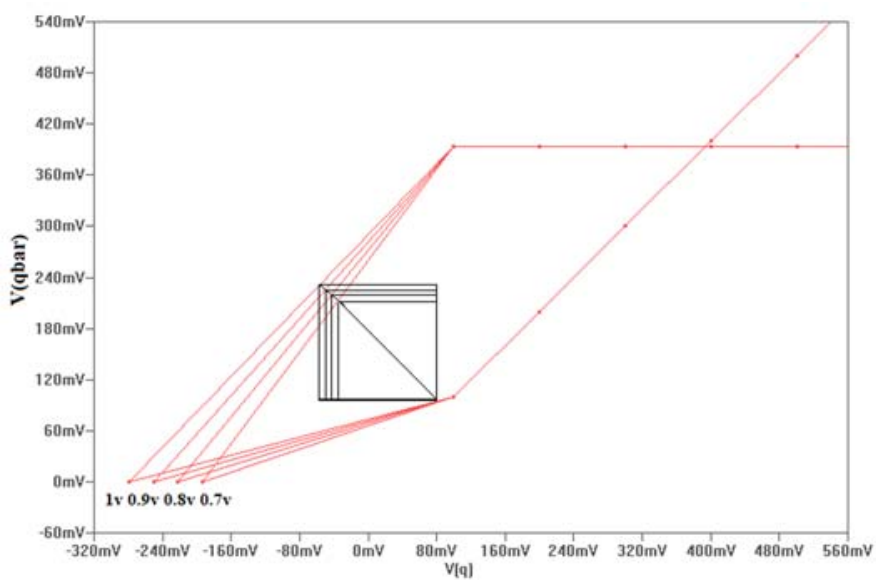

Fig. 9. VTC of proposed 8T SRAM with varying Vdd.

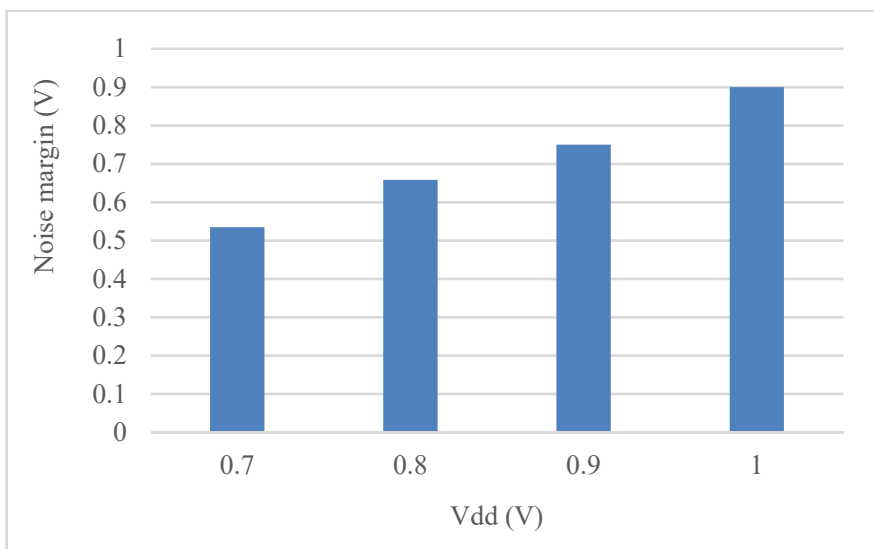

Fig. 10. Noise Margin of proposed 8T SRAM with varying Vdd.

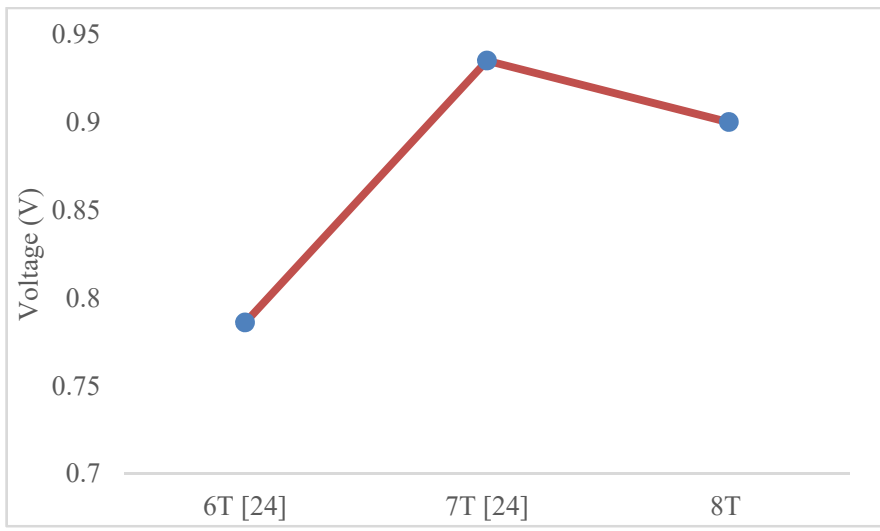

Fig. 11. Comparison of Noise Margin among conventional SRAMs and proposed 8T SRAM.

Figure 11 demonstrates that the calculated SNM of the proposed cell along with several other conventional memory cells. This assessment is done with $45 \mathrm{~nm}$ CMOS technology for the entire memory cell (6T, $7 \mathrm{~T}$ and proposed 8T SRAM) where the supply voltage was considered $1 \mathrm{~V}$. However, the proposed cell shows higher amount of noise margin than $6 \mathrm{~T}$ SRAM and significantly $0.35 \mathrm{v}$ less noise margin than $7 \mathrm{~T}$ SRAM. With a better noise margin proposed cell helps to save the stored data from external noise in the system.

\section{F. Leakage Current Analysis of Proposed Design:}

The write ability of a SRAM is determined by applying ' 1 ' to BL and ' 0 ' to BLB. Cell current or write current (Iwrite) can be expressed analytically by solving Kirchoff's current law (KCL) [22] at storage node Q which is storing ' 1 '. Where current is flowing through M5, M1 and leaving through M3 (figure 12). For node Q the KCL is -

$$
\text { Iwrite }=\operatorname{Im} 5+\operatorname{Im} 1-\operatorname{Im} 3
$$

where,

$\operatorname{Im} 1, \operatorname{Im} 3$ and $\operatorname{Im} 5$ are the currents following through the transistors M1, M3 and M5 respectively.

$$
\begin{aligned}
& \operatorname{Im} 5\left(V g s=V_{W R L}-V_{Q} ; V d s=V_{B L}-V_{Q}\right) \\
& \text { Im1 }\left(V g s=V_{Q} ; V d s=V_{Q}-V d d\right) \\
& \text { Im3 }\left(V g s=V_{Q B} ; V d s=V_{Q}\right)
\end{aligned}
$$

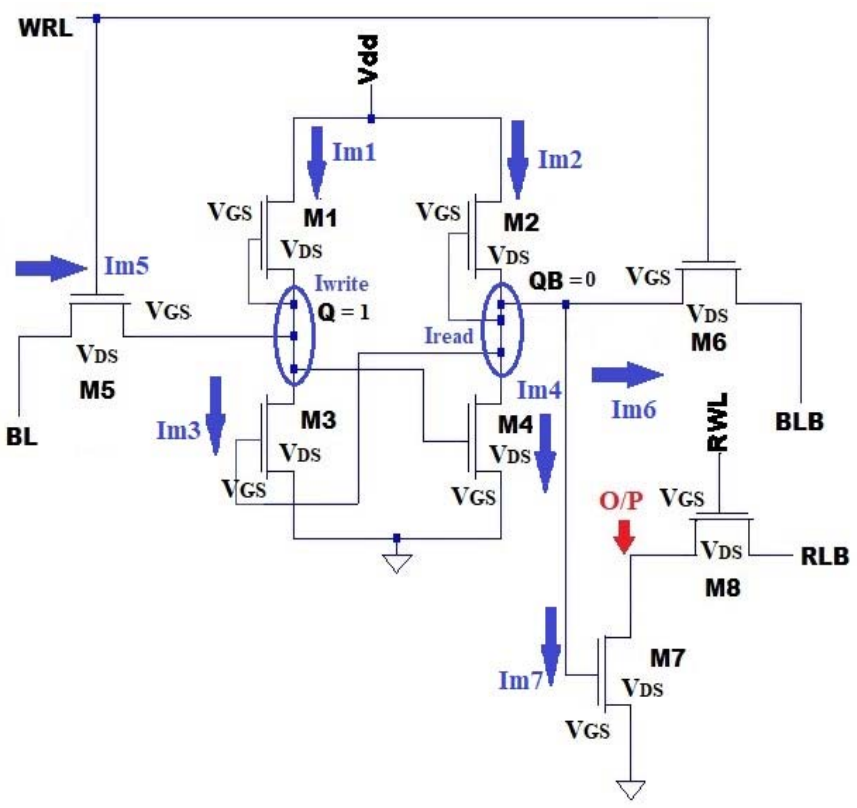

Fig. 12. Current passing through proposed 8T SRAM.

For read ability the Kirchoff's current law is applied to the complimentary storage node QB. The current is passing through M2, M6, M8 and leaving through M4 and M8 (figure 12). For node $\mathrm{QB}$ the $\mathrm{KCL}$ is -

$\operatorname{Iread}=\operatorname{Im} 2-\operatorname{Im} 4-\operatorname{Im} 6-\operatorname{Im} 7$.

where,

$\operatorname{Im} 2, \operatorname{Im} 4, \operatorname{Im} 6$ and $\operatorname{Im} 7$ are the currents following through the transistors M2, M4, M6 and M7 respectively.

$$
\begin{aligned}
& \operatorname{Im} 2\left(V g s=V_{Q B} ; V d s=V_{Q B}-V d d\right) \\
& \operatorname{Im} 4\left(V g s=V_{Q} ; V d s=V_{Q B}\right) \\
& \operatorname{Im} 6\left(V g s=V_{W R L}-V_{Q B} ; V d s=V_{B L B}-V_{Q B}\right) \\
& \operatorname{Im} 7\left(V g s=V_{Q B} ; V d s=V_{o / p}\right)
\end{aligned}
$$

While calculating the leakage current in standby mode or static mode the word lines (WRL and RWL) are deactivated and $\mathrm{V}_{\mathrm{t}}$ was considered as $0.3 \mathrm{~V}$. For this proposed 8T SRAM, the operating mode, gate to source voltage (Vgs), drain to source voltage $(\mathrm{Vds})$ are reported to Table III. 


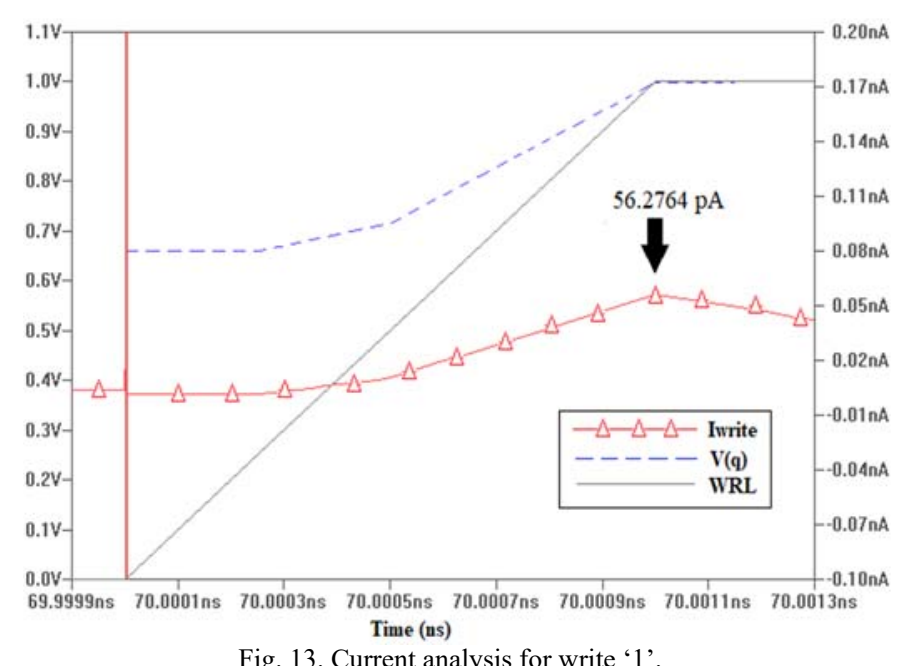

As there are three basic regions of operation for a MOSFET, they are cutoff, linear and saturation. In cut off region no current can flow through. From figure 12 and Table III, transistors M1, M3, M6 and M7 stay in the cutoff region (Vgs $<\mathrm{Vt}$ ), where the gate to source voltage of those transistor is less than the threshold voltages. Which mean no current can flow through this transistor in static mode. Transistor M4 stays in the linear zone $(\mathrm{Vds}<\mathrm{Vgs}-\mathrm{Vt})$. In this region the gate to source voltage of M4 is less than threshold voltage. Which means current flows from drain to source and the current passing through it is roughly proportional to both $\mathrm{Vgs}$ and Vds. Lastly, transistors M2, M5 and M8 stay in saturation region ( $\mathrm{Vds}>\mathrm{Vgs}$ or, $\mathrm{Vds}+\mathrm{Vt}>\mathrm{Vgs}$ ) where the amount of flowing current is proportional to the square of $\mathrm{Vgs}$, and is independent of $\mathrm{Vds}$.

Results are plotted in figure 13 for write current. And roughly $56.2764 \mathrm{pA}$ when the storage node $\mathrm{Q}$ is storing ' 1 '. The voltage and current is changing their state proportionally for writing data whereas, it shows inversely proportion phenomenon while reading data. By solving KCL equation (2) for reading data, the current shows a negative value of $1.8994 \mathrm{pA}$ at the point where the read output shows around $997.93839 \mathrm{mV}$ (figure 14).

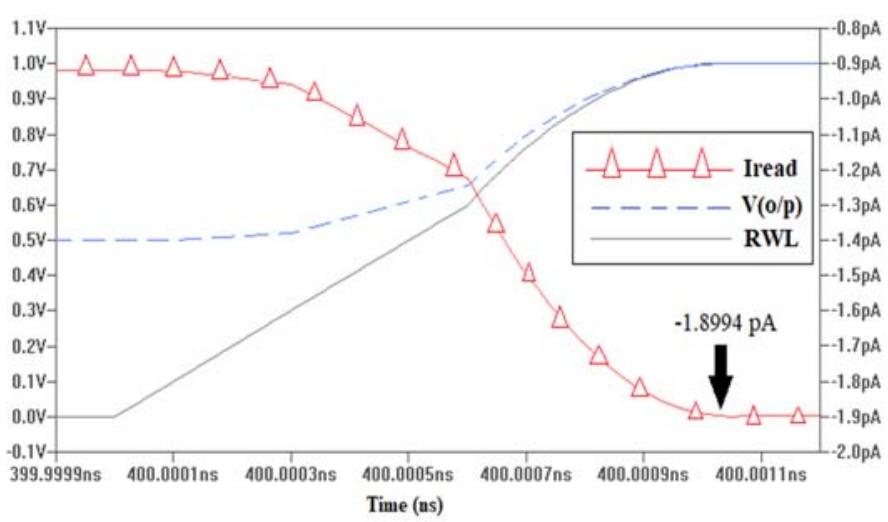

Fig. 14. Current analysis for read ' 1 '.

\section{G. The effect of process corner analysis:}

Process variation rises from a number of factors, which includes discrete random dopants, line edge roughness, oxide thickness, etc. [23]. The fabrication process of IC (integrated circuit) is maiden cause of process variation. The process variation turn into dominant factor due to increase number of scaling which eventually mismatch the components. Hence, for SRAM this mismatch is very decisive [13]. Sometimes the design parameters like channel length and threshold voltage affects the process variation by enhancing the performance of circuit. A corner based approach is preferred to find the effect of process variations on SRAM performance. In our proposed model we have used all nMOS transistors with different CMOS technology where the pull up transistors (M1, M2), access transistors (M5, M6) and pull down transistors (M3, M4) are chosen as $32 \mathrm{~nm}, 45 \mathrm{~nm}$ and $65 \mathrm{~nm}$. The corner analysis has been done according to variation of lengths.

Figure 15, illustrates the dissimilarity of stored data for highest $120^{\circ} \mathrm{C}$ temperature variation at each process corner (FF, FS, TT, SF, and SS) with respect to typical process corner at nominal $27^{\circ} \mathrm{C}$ temperature. All the Qbar curves stores around $500 \mathrm{mV}$ at $\mathrm{Q}=0 \mathrm{~V}$. It has been clearly seen that the highest curve was achieved by SS process corner and the lowest curve was achieved by FF process corner. Here SS corner indicates both the pull up transistors and pull down transistors are slow and the decline rate is sluggish. Nevertheless, FF corner appearances with the vice versa result of SS corner having both fast operated pull up transistors and pull down transistors. The TT process corner resides exactly on the middle, so the effect of the transistors are moderate as they are in their typical mode. The SF process corner stays between the TT and FF process corner. At this corner the pull up transistors is stronger than pull down transistors. The last FS process corner takes the bottom position between TT and SS where the pull down transistors are much more dominant than the pull up transistors. While comparing the process corners of $120^{\circ} \mathrm{C}$ with nominal temperature $27^{\circ} \mathrm{C}$, we can see that the declining rate of nominal temperature was sharp slope than the other curves which stay above the nominal curve. At $\mathrm{Q}=1 \mathrm{~V}$ all the corner curves of $120^{\circ} \mathrm{C}$ resided at $200 \mathrm{mV}$ as Qbar whereas the nominal curve resided at $0 \mathrm{~V}$. So the voltage difference of Qbar between temperature $120^{\circ} \mathrm{C}$ and nominal temperature $27^{\circ} \mathrm{C}$ was around $120 \mathrm{mV}$.

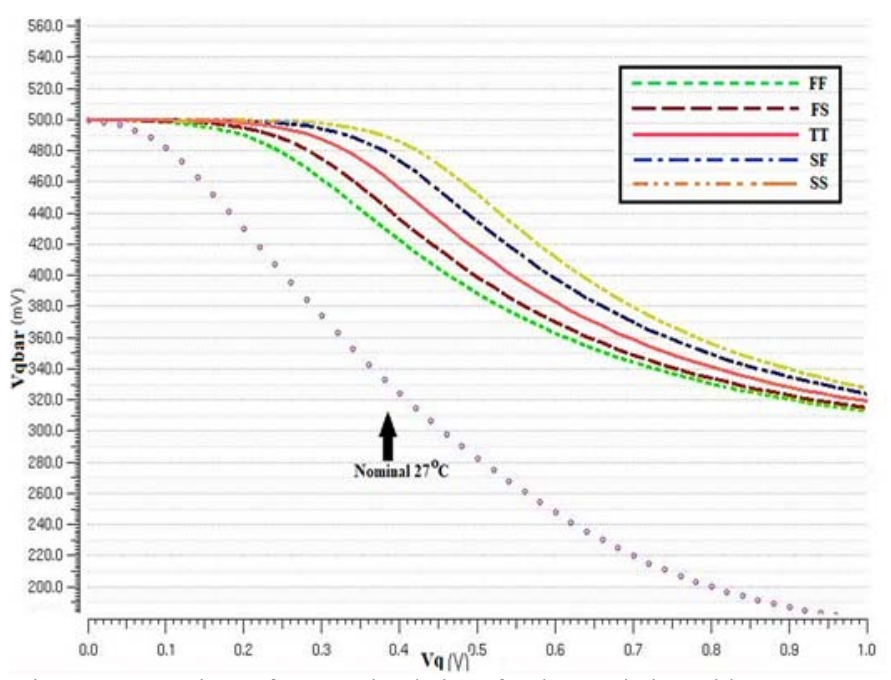

Fig. 15. Comparison of corner simulations for data variation with respect to temperature $120^{\circ} \mathrm{C}$ and nominal temperature $27^{\circ} \mathrm{C}$. 
Another process corner deviation of stored voltage was shown in figure 16 . In this case the comparison was done by lowest temperature $-10^{\circ} \mathrm{C}$ and nominal temperature $27^{\circ} \mathrm{C}$. All the curve of $-10^{\circ} \mathrm{C}$ located below the nominal temperature. As previous, all the curves stores $500 \mathrm{mV}$ as Qbar when Q stores $0 \mathrm{~V}$ but it ended at different voltage level at $\mathrm{Q}=1 \mathrm{~V}$.

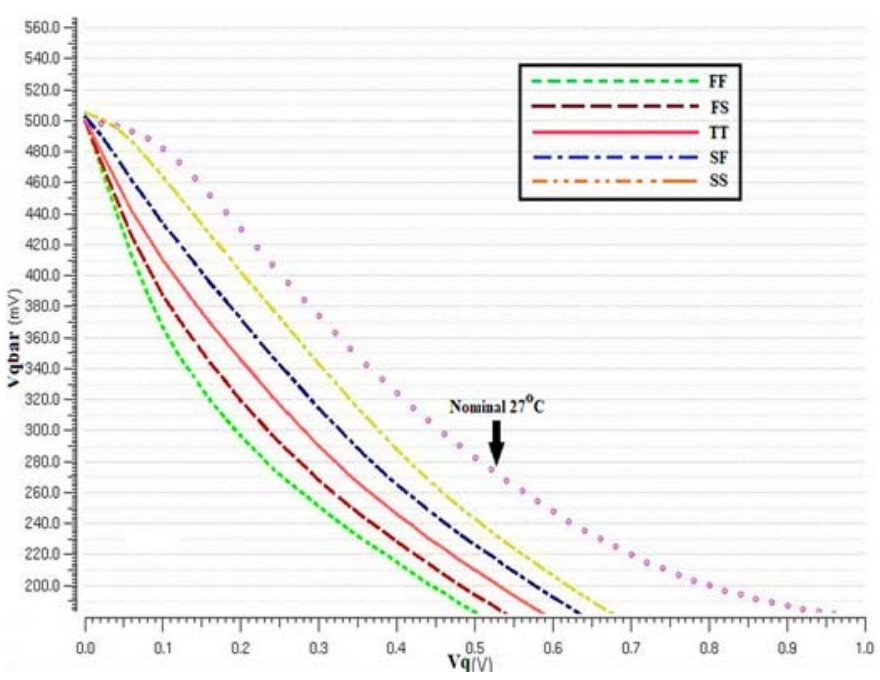

Fig. 16. Comparison of corner simulations for data variation with respect to temperature $-10^{\circ} \mathrm{C}$ and nominal temperature $27^{\circ} \mathrm{C}$.

Here the similarity in variation of process corner is displayed according to $120^{\circ} \mathrm{C}$. likewise, the decay rate is high for SS process corner and lowest for FF process corner. Compared to the nominal $27^{\circ} \mathrm{C}$ and the temperature $-10^{\circ} \mathrm{C}$, the variation of stored voltage $\mathrm{Q}$ was approximately $0.3 \mathrm{~V}, 0.35 \mathrm{~V}, 0.4 \mathrm{~V}$, $0.45 \mathrm{~V}$ and $0.5 \mathrm{~V}$ for SS, SF, TT, FS and FF consecutively at the point $190 \mathrm{mV}$. So varying the temperature from positive temperature $120^{\circ} \mathrm{C}$ to negative temperature $-10^{\circ} \mathrm{C}$, the $\mathrm{Q}$ vs Qbar curve at each process corner shows the same trend as the nominal temperature. Conversely with the increase of temperature the decline rate of process variation decreases. This validates the temperature analysis at different process corner performed here.

\section{H. Area Comparison:}

The layouts of proposed 8T SRAM cell is drawn in Cadence design tool (Figure 17 and 18) for both $45 \mathrm{~nm}$ and $90 \mathrm{~nm}$ technology. Table IV illustrates the comparison of area among 6T SRAM [2], 7T SRAM [24] and 8T SRAM [27] in according to the transistor's length $90 \mathrm{~nm}$ technology. Yet again proposed cell occupies area of $2.19 \mu \mathrm{m}^{2}$ for $45 \mathrm{~nm}$ length. Where it is visibly seen that the proposed cell consumes less area compared to other conventional models. Due to the absence of pMOS in the proposed cell, the total area drastically drops down.

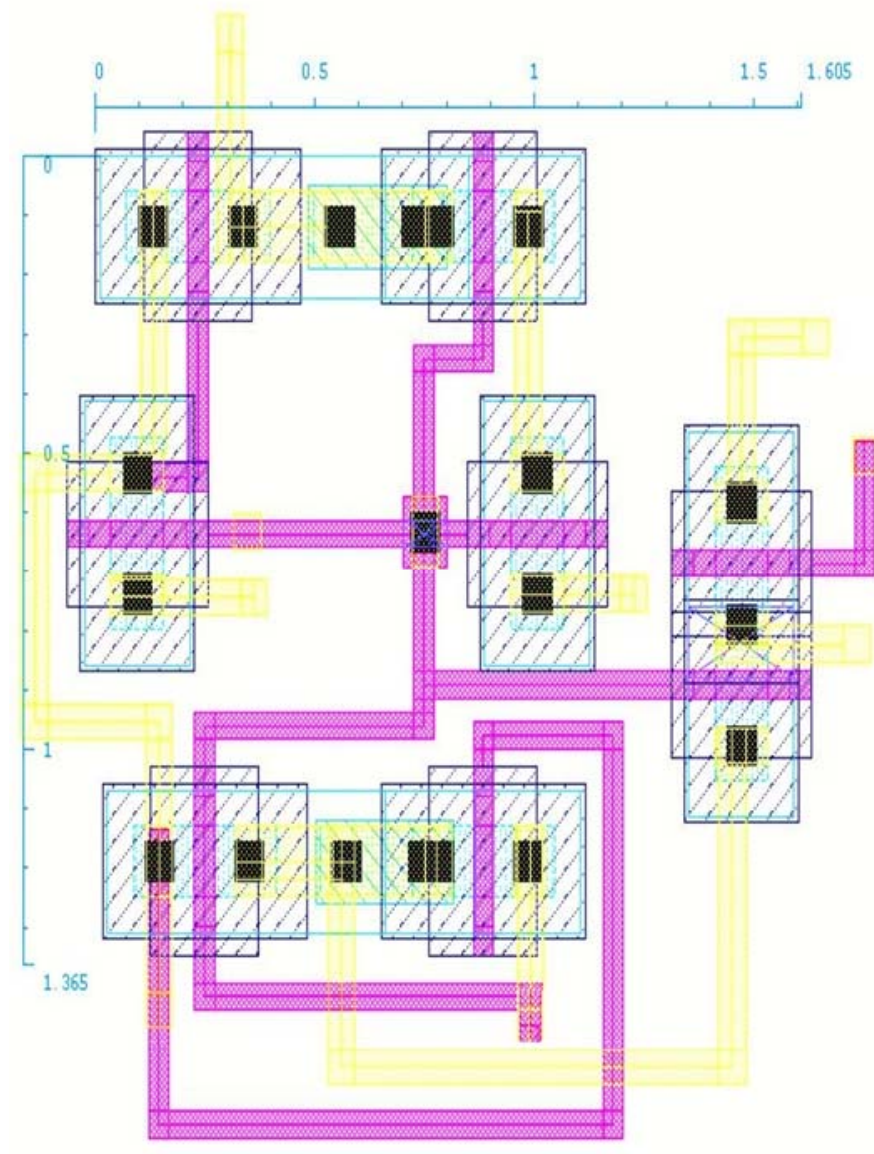

Fig. 17. Layout of proposed 8T SRAM cell of $45 \mathrm{~nm}$.

Table IV

COMPARISON OF AREA In ACCORDING TO LENGTH OF THE TRANSISTORS

\begin{tabular}{ccccc}
\hline \hline $\begin{array}{c}\text { Length of } \\
\text { transistors }\end{array}$ & 6T SRAM & 7T SRAM & 8T SRAM & $\begin{array}{c}\text { Proposed } \\
\text { 8T SRAM }\end{array}$ \\
\hline $90 \mathrm{~nm}$ & $12.35 \mathrm{~mm}^{2}$ & $11.65 \mathrm{um}^{2}$ & $11.78 \mu \mathrm{m}^{2}$ & $7.048 \mathrm{um}^{2}$ \\
& {$[2]$} & {$[24]$} & {$[27]$} & \\
\hline \hline
\end{tabular}

\section{CONCLUSION}

A new memory model of 8T SRAM cell is proposed in this paper by some useful amount of simulation survey. The primary factor of this proposed cell is it only takes nMOS transistors instead of pMOS transistors to build a static RAM. Thus the overhead area and consumption of power is comparatively less $4.7 \mathrm{um}^{2}$ and $1.9 \mathrm{pW}$ than conventional $8 \mathrm{~T}$ SRAM. Despite of having more transistors equated with conventional 6T and 7T SRAM the propose cell shows better read time delay and improved noise margin. Alongside with the speed the developed cell prevents the most common stability problem of memory cell and performs overwrite and successive read operation without any fluctuation. With lowest operating voltage of $0.7 \mathrm{Vdd}$, the proposed cell can be reflected as a farm contender for the memory used in system on chip integrated circuits. 


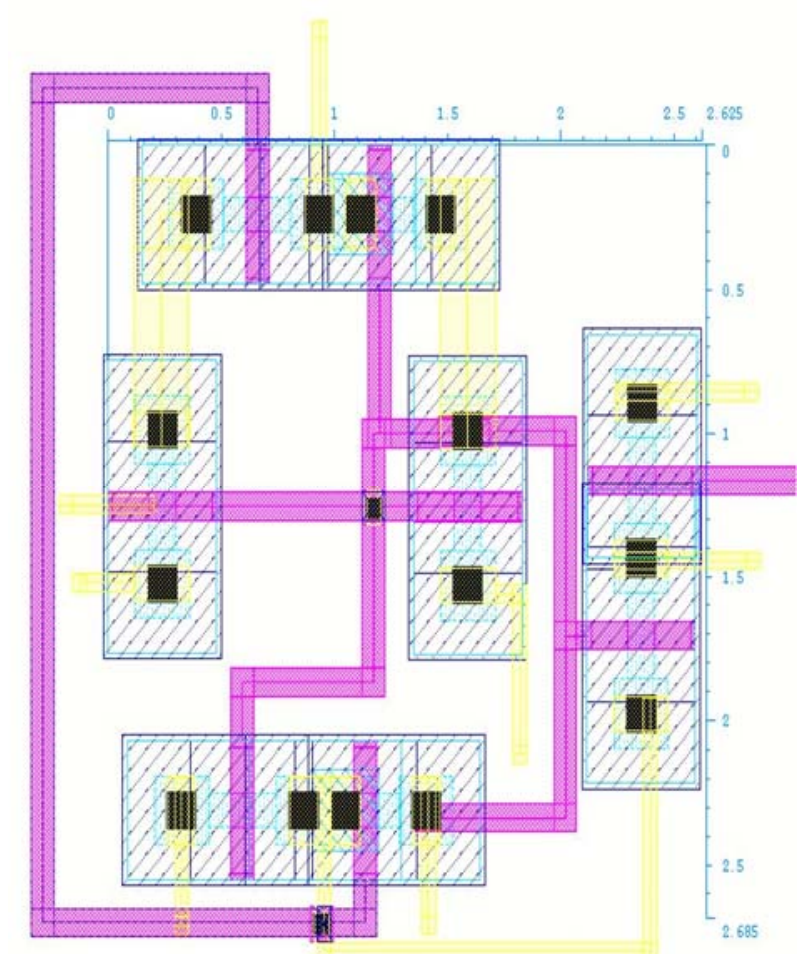

Fig. 18. Layout of proposed $8 \mathrm{~T}$ SRAM cell of 90nm.

\section{REFERENCES}

[1] S. Robert, Technological innovation in the semiconductor industry: a case study of the International Technology Roadmap for Semiconductors (ITRS). Diss. George Mason University, 2004.

[2] A. Paridhi, and S. Dasgupta, "A Comparative Study of 6T, 8T and 9T Decanano SRAM cell." Industrial Electronics \& Applications, 2009. ISIEA 2009. IEEE Symposium on. Vol. 2. IEEE, 2009.

[3] A. Asen, A. R. Brown, J. H. Davies, S. Kaya, and G. Slavcheva. "Simulation of intrinsic parameter fluctuations in decananometer and nanometer-scale MOSFETs." IEEE transactions on electron devices 50, no. 9 (2003): 1837-1852.

[4] M. Tomohisa, J. Okumtura, and A. Toriumi. "Experimental study of threshold voltage fluctuation due to statistical variation of channel dopant number in MOSFET's." IEEE Transactions on Electron Devices 41, no. 11 (1994): 2216-2221.

[5] G. F. Cardinale, et al. "Demonstration of pattern transfer into sub-100 $\mathrm{nm}$ polysilicon line/space features patterned with extreme ultraviolet lithography." Journal of Vacuum Science \& Technology B: Microelectronics and Nanometer Structures Processing, Measurement, and Phenomena 17.6 (1999): 2970-2974.

[6] J. Singh, D. K. Pradhan, S. Hollis, \&, S. P. Mohanty (2008), "A single ended 6T SRAM cell design for ultra-low-voltage applications". IEICE Electronics Express, 5(18), 750-755.

[7] H. Mizuno, \& T. Nagano, (1996). "Driving source-line cell architecture for sub-1-V high-speed low-power applications". IEICE transactions on electronics, 79(7), 963-968.

[8] Z. Bo, et al. "A Sub-200mV 6T SRAM in 0.13um CMOS in Solid-State Circuits Conference," 2007. ISSCC 2007. Digest of Technical Papers. IEEE International. 2007.

[9] A. Touqeer, B. Cheng, and D.R. Cumming. "Variability resilient lowpower 7T-SRAM design for nano-scaled technologies." Quality Electronic Design (ISQED), 2010 11th International Symposium on. IEEE, 2010.
[10] K. F. Sharif, R. Islam, M. Haque. M. A. Keka, \& S. N. Biswas, (2017, February). "7T SRAM based memory cell". In Innovative Mechanisms for Industry Applications (ICIMIA), 2017 International Conference on (pp. 191-194). IEEE.

[11] M. Moghaddam, M. H. Moaiyeri, \& M. Eshghi, (2015, May). "Ultra low-power 7T SRAM cell design based on CMOS". In Electrical Engineering (ICEE), 2015 23rd Iranian Conference on (pp. 1357-1361). IEEE.

[12] P. Macken, M. Degrauwe, M. Van Paemel, \&, H. Oguey (1990, February). "A voltage reduction technique for digital systems". In Solid-State Circuits Conference, 1990. Digest of Technical Papers. 37th ISSCC, 1990 IEEE International (pp. 238-239). IEEE.

[13] R. Gupta, \&, S, Dasgupta. (2017). "Process Corners Analysis of Data Retention Voltage (DRV) for 6T, 8T, and 10T SRAM Cells at $45 \mathrm{nm".}$ IETE Journal of Research, 1-6.

[14] Anh-Tuan, Do, et al. "An 8T differential SRAM with improved noise margin for bit-interleaving in $65 \mathrm{~nm}$ CMOS." IEEE Transactions on Circuits and Systems I: Regular Papers 58.6 (2011): 1252-1263.

[15] M. Yabuuchi, K. Nii, Y Tsukamoto, S. Ohbayashi, Y. Nakase, \& H. Shinohara, (2009, June). "A 45nm 0.6 V cross-point 8T SRAM with negative biased read/write assist." In VLSI Circuits, 2009 Symposium on (pp. 158-159). IEEE.

[16] http://ptm.asu.edu

[17] J. M. Rabaey, A. Chandrakasan, and B. Nikolic, "Digital Integrated Circuits:A Design Perspective", 2nd ed. New Delhi, India: PrenticeHall, 2005.

[18] P. Raikwal, V. Neema, \& A. Verma, (2017, April). "High speed 8T SRAM cell design with improved read stability at $180 \mathrm{~nm}$ technology". In Electronics, Communication and Aerospace Technology (ICECA), 2017 International conference of (Vol. 2, pp. 563-568). IEEE.

[19] K. F. Sharif, R. Islam, S. N. Biswas, \& V. Groza. (2017, April). "4 Transistor and 2 memristor based memory". In Computer Applications \& Industrial Electronics (ISCAIE), 2017 IEEE Symposium on (pp. 3740). IEEE.

[20] C. Benton and A. P. Chandrakasan. "Static noise margin variation for sub-threshold SRAM in 65-nm CMOS." IEEE Journal of solid-state circuits 41.7 (2006): 1673-1679.

[21] K. F. Sharif, R. Islam, M. Haque, S. N. Biswas, V. Groza, \& M. Assaf, (2017, February). "Low power nMOS based memory cell". In Innovative Mechanisms for Industry Applications (ICIMIA), 2017 International Conference on (pp. 186-190). IEEE

[22] K.F. Sharif, R. Islam and S.N. Biswas, "Low Power Novel 10T SRAM with Stabled Optimized Area." In 2018 IEEE International WIE Conference on Electrical and Computer Engineering (WIECON-ECE), pp. 21-24. IEEE, 2018.

[23] E. Seevinck, F. List, J. Lohstroh, "Static noise margin analysis of MOS SRAM cells", IEEE J. Solid-State Circuits, vol. SC-22, no. 5, pp. 748754, Oct. 1987.

[24] K.F. Sharif, R. Islam and S.N. Biswas, "A New Model of High Speed 7T SRAM Cell". In 2018 International Conference on Computer, Communication, Chemical, Material and Electronic Engineering (IC4ME2) (pp. 1-4). IEEE. 2018, February

[25] A. Islam, and M. Hasan. "A technique to mitigate impact of process, voltage and temperature variations on design metrics of SRAM Cell." Microelectronics Reliability 52.2 (2012): 405-411.

[26] C. Binjie, et al. "Impact of intrinsic parameter fluctuations in decanano MOSFETs on yield and functionality of SRAM cells." Solid-State Electronics 49.5 (2005): 740-746.

[27] S. Abhijit, S. Ghosh, and M. Bayoumi. "A novel 90nm 8T SRAM cell with enhanced stability." Integrated Circuit Design and Technology, 2007. ICICDT'07. IEEE International Conference on. IEEE, 2007. 